Research Article

\title{
Compressed Sensing Algorithm-Based Magnetic Resonance Imaging Guided for Prognosis of Early Neurological Deterioration after Intravenous Thrombolysis in Ischemic Stroke Patients
}

\author{
Yiwei Huang $\mathbb{D}$, Xiaoyun Sun $\mathbb{D}$, Yinping Yao $\mathbb{D}$, Yejun Chen $\mathbb{D}$, Yan Chen $\mathbb{D}$, \\ and Yuping $\mathrm{He} \mathbb{D}$
}

Department of Neurology, Zhuji People's Hospital, Zhuji City 311800, Zhejiang Province, China

Correspondence should be addressed to Yuping He; 1651080363@xzyz.edu.cn

Received 20 May 2021; Accepted 26 June 2021; Published 6 July 2021

Academic Editor: Gustavo Ramirez; drgustavophd@gmail.com

Copyright (C) 2021 Yiwei Huang et al. This is an open access article distributed under the Creative Commons Attribution License, which permits unrestricted use, distribution, and reproduction in any medium, provided the original work is properly cited.

\begin{abstract}
This work was aimed to study the risk factors and prognostic treatment for acute ischemic stroke (AIS) patients with early neurological deterioration (END) after intravenous thrombolytic therapy via compressed sensing algorithm-based magnetic resonance imaging (CS-MRI). 231 patients who were diagnosed with AIS were selected, and the final involved number of patients was 182. Patients with AIS were treated with intravenous thrombolysis with alteplase within 4.5 hours of onset. After treatment, patients with early neurological deterioration were defined as the deteriorating group and those without early neurological impairment were defined as the nondeteriorating group. In univariate analysis, hypertension, white blood cell count, and National Institutes of Health Stroke Scale (NIHSS) score were correlated with the occurrence of END. Under the CS-MRI theory, the two groups of patients were evaluated for middle cerebral artery basal ganglia infarction and internal watershed infarction. After univariate analysis, the $P<0.1$ variables were taken as the independent variable, and the binary logistic regression model was adopted for multivariate regression analysis. It was disclosed that NIHSS score was not correlated with the occurrence of early neurological function deterioration, while homocysteine was. Hypertension, white blood cell count, homocysteine, and NIHSS score were risk factors for END. The image analysis revealed that the incidence of deteriorating basal ganglia infarction group was lower relative to the nondeteriorating group, and the incidence of watershed infarction was higher in the deteriorating group versus the nondeteriorating group. The image analysis suggested that predicting the occurrence of END through risk factors can actively provide endovascular treatment for patients with AIS.
\end{abstract}

\section{Introduction}

AIS, also known as cerebral infarction, refers to the stenosis or occlusion of the blood supply arteries of the brain and is the general term for brain tissue necrosis caused by insufficient blood supply to the brain [1]. END after intravenous thrombolytic therapy for AIS refers to neurological function of patients with stroke, after intravenous thrombolytic therapy improves in a short period of time and then fluctuates or deteriorates in a stepped manner [2]. END indicates a longterm poor prognosis in patients with AIS. So far, few literatures have pointed out predictive indicators and methods for END.
At present, medical imaging technology is developing rapidly, and MRI technology has been widely adopted in the evaluation of various diseases [3]. In actual diagnosis, the traditional basic particle swarm algorithm needs to collect a large amount of data, which prolongs the time of data collection, and the influence of noise and artifacts will interfere with the image [4]. Therefore, reducing the number of data collection, reducing collection time, and forming streamlined and fast MRI images have become the major problems that modern medicine continues to solve. Some studies have proposed that compressed sensing theory is currently one of the hot topics in the field of information engineering [5], which 
breaks the traditional sampling theory. Compared with the minimum sampling frequency, the CS theory can recover the original signal from lower sampling data with high probability while maintaining the normal retention of data information, and the data collection time and data processing time are reduced. Therefore, the compressed sensing theory is widely utilized in reconstructing MRI images, and some studies have proposed the CS-MRI theory [6]. In the actual application of MRI in medicine, the patient's heart, pulse, and other organs will move slightly during the scanning process, which will cause phase disturbance in the MRI system during data acquisition, resulting in phase error, and the constructed MRI image will be interfered by motion artifacts. Moreover, noise is prone to be generated during data acquisition, resulting in amplitude noise in the measurement data. Phase error and amplitude noise will make the constructed MRI image lack clarity and affect the final clinical diagnosis [7]. Based on the CS-MRI algorithm, under the conditions of total variation and adaptive tight frame, the relative error generated by the measurement data was corrected and the amplitude noise was removed, to reconstruct the highquality MRI images.

In summary, CS-MRI assessment for END patients was made to explore the risk factors of END and reduce the incidence of END by controlling the risk factors. If END can be predicted early based on the patient's clinical data, corresponding treatment measures can be implemented to reduce the neurological damage caused by END to the patient, thereby improving the prognosis of patients with END, which was of great clinical value for the later exploration of END.

\section{Materials and Methods}

2.1. Selection of Research Objects. 231 patients were selected, who were diagnosed with AIS and met the diagnostic criteria in hospital from September 15, 2017, to August 5, 2020. According to the exclusion criteria, it was finally determined that 182 cases were selected, 75 patients who received intravenous thrombolytic therapy with early functional deterioration were defined as the deterioration group, and 107 patients without early functional deterioration were defined as the nondeteriorating group. The study was approved by the Medical Ethics Committee of Hospital, and the patients and their families understood the research situation and signed an informed consent form.

Inclusion criteria were as follows: (I) patients met the AIS diagnostic criteria, (II) the onset time was less than $4.5 \mathrm{~h}$, (III) the patient underwent CT examination without intracranial hemorrhage, and (IV) patients received intravenous thrombolysis within $4.5 \mathrm{~h}$ of onset.

Exclusion criteria were as follows: (I) patients with neurological impairment caused by epilepsy symptoms, (II) patients with heart, liver, kidney, and other related diseases at the same time, (III) pregnant women, and (IV) patients with intracranial tumors or arterial tumor.

2.2. Basic Data Collection. Clinical data: age, gender, blood pressure, past history (hypertension, diabetes, coronary heart disease, heart failure, smoking, drinking, myocardial infarction, history of antiplatelet, or lipid-lowering drugs). NIHSS score: before thrombolysis and 4.5 hours, 24 hours, and 1 week after thrombolysis. Laboratory test results' data: white blood cell count, emergency blood glucose ( $\mathrm{mmol} / \mathrm{L})$, platelet count, International Normalized Ratio (INR), lowdensity lipoprotein, high-density lipoprotein, fibrinogen, and homocysteine $(\mu \mathrm{mol} / \mathrm{L})$.

2.3. Experimental Evaluation Methods. The diagnosis of AIS referred to the diagnostic criteria in the "Chinese Guidelines for the Diagnosis and Treatment of Acute AIS 2014." Neurological impairment was assessed using the NHISS. Patients were given baseline NIHSS scores immediately after admission, and NIHSS scores were given immediately at 4.5 hours, 24 hours, and 1 week after thrombolysis. Within 24 hours, after intravenous thrombolysis, was the optimal treatment time for patients, which was of great significance to the prognosis of patients. The NIHSS score is widely utilized in the evaluation of patients with acute AIS. In the NIHSS scoring, the reliability of each individual item score is better, but the overall total score is quite different. To avoid the result of NIHSS score variation, $\triangle$ NIHSS $\geq 2$ points are taken as the occurrence of general END. END is the fluctuating or stepwise deterioration of patients after intravenous thrombolysis with alteplase, and the baseline NIHSS score is a diagnostic criterion for evaluating the occurrence of END. To improve the accuracy of diagnosis, different baseline NIHSS scores and different END diagnostic criteria were adopted. If the baseline NIHSS score was less than 6 points, it was recorded as an increase of 2 points or more after intravenous thrombolysis within 24 hours. If the baseline NIHSS score was $\geq 6$ points, it was recorded as an increase of 4 points or more after intravenous thrombolysis within 24 hours.

2.4. Phase Error Model of CS-MRI. When the phase error model collects data, it is necessary to transform the MRI images with a certain order into the frequency domain and then sample in the frequency domain ( $S$-space) data. Fourier transform expressions of MRI image domain data and frequency domain data are as follows:

$$
F\left(S_{x}, S_{y}\right)=\iint V(x, y) e^{-i 2 \pi\left(x S_{x}+y S_{y}\right)} \mathrm{d} x \mathrm{~d} y .
$$

In (1), the $S_{x}$ direction is the frequency encoding direction of the $S$-space, and the $S_{y}$ direction is the readout direction of the $S$-space. $F\left(S_{x}, S_{y}\right)$ is the data of the $S$-space coordinates $\left(S_{x}, S_{y}\right)$, and $V(x, y)$ is the density function of the image domain.

The frequency domain data expression of the MRI image with the phase error is as follows:

$F\left(S_{x}, S_{y}\right)=\iint V\left(x-g\left(s_{y}\right), y-h\left(s_{y}\right)\right) e^{-i 2 \pi\left(x S_{x}+y S_{y}\right)} \mathrm{d} x \mathrm{~d} y$.

In equation (2), $g\left(S_{y}\right)$ and $h\left(S_{y}\right)$ are moving distance of image density functions $V(x, y)$ in the $x$ direction and the $y$ direction, respectively. 
It is assumed that $\hat{x}=x-g\left(S_{y}\right)$ and $\hat{y}=y-h\left(S_{y}\right)$, which are substituted in equations (2) and (3) and are then acquired:

$\widehat{F}\left(S_{x}, S_{y}\right)=\iint V(\widehat{x}, \hat{y}) e^{-i 2 \pi\left[S_{x}\left(\widehat{x}+g\left(S_{y}\right)\right)+S_{y}\left(\hat{y}+p\left(S_{y}\right)\right)\right]} \mathrm{d} \widehat{x} \mathrm{~d} \hat{y}$.

Through the contrast of equations (2) and (3), equation (4) is obtained:

$$
\widehat{F} \otimes\left(S_{x}, S_{y}\right)=e^{-i \phi\left(S_{x}, S_{y}\right)} F\left(S_{x}, S_{y}\right) .
$$

In equation (4), $\Phi\left(S_{x}, S_{y}\right)=2 \pi S_{x} g\left(S_{y}\right)+S_{y} g\left(S_{y}\right)$, and it represents the phase error of $S$-space data in MRI images.

2.5. Evaluation of CS-MRI Scan. MR Prisma 3.0 magnetic resonance instrument produced by Siemens (Germany) was utilized to evaluate patients. During the scan, let the patient lie supine, keep breathing smoothly, tilt the head slightly back, and fully expose the neck. The linear array probe scanned the common carotid artery from the proximal end to the distal end, and the examination procedure was described to the patient. Scanning parameters: the matrix was $251 \times 251$, the layer thickness was $3.5 \mathrm{~mm}$, the field of view was $25 \times 25 \mathrm{~cm}$, the flip angle was $15^{\circ}$, and the layer spacing was $6.1 \mathrm{~mm}$. AIS location in the blood supply area of the middle cerebral artery: basal ganglia infarction, internal watershed infarction, and regional infarction.

2.6. Statistical Methods. SPSS24.0 statistical software was adopted to calculate and analyze the data. The measurement data conforming to the normal distribution were represented by the mean \pm standard deviation $(\bar{x} \pm s)$, and the nonconforming ones were represented by the percentage (\%). In univariate analysis, the variable with $P<0.1$ was analyzed by the binary logistic regression analysis model, and then, the multivariate regression analysis was conducted. Finally, the relevant independent predictors were determined, and the odds' ratio (OR) and $95 \%$ confidence interval (CI) were calculated. $P<0.05$ indicated the difference was statistically significant.

\section{Results}

3.1. Statistics of Patient END Occurrence Time. From September 15, 2019, to August 5, 2020, among the 231 AIS patients who were treated, they were excluded from bridging treatment, and the number of selected patients was finally determined to be 182 . Intravenous thrombolysis was performed 4.5 hours after admission. After intravenous thrombolysis, there were 22 cases of END patients and 160 cases of nondeteriorating patients, and the incidence of END was $12.1 \%$.

According to the diagnostic criteria of END, they were divided into the deteriorating group and the nondeteriorating group. There were 22 cases $(12.1 \%)$ in the deteriorating group, including 14 males $(63.6 \%)$ and $8 \mathrm{fe}-$ males $(36.4 \%)$, with an average age of $65.9 \pm 10.7$ years, and
160 cases in the nondeteriorating group, 125 males (78.1\%) and 35 females (21.9\%), with an average age of $62.7 \pm 9.5$ years; $P>0.05$, and the difference was not obvious. After the thrombolytic therapy, there were differences in the time of occurrence of END in patients (Figure 1). END occurred in 8 cases within $24 \mathrm{~h}$ and 9 cases from $24 \mathrm{~h}-48 \mathrm{~h}$. It can be clearly seen that it was concentrated within $24 \mathrm{~h}$ and $24-48 \mathrm{~h}$, that is, the occurrence time of patients' END was mainly within $48 \mathrm{~h}$ after admission and $72 \mathrm{~h}$ after stroke (77.3\%).

\subsection{Contrast of Characteristics of Deteriorating Patients and} Nondeteriorating Patients. A total of 182 patients were enrolled, 22 of which developed END and 160 did not. The characteristics of the two groups of patients were compared in Figure 2, and it was found that hypertension and white blood cell count were correlated with the occurrence of END, and the difference was notable, $P<0.05$. Diabetes, coronary heart disease, smoking, drinking, platelet count, international standardized ratio, low-density lipoprotein, high-density lipoprotein, and fibrinogen all showed $P>0.05$, suggesting no evident difference, as shown in Figure 3.

\subsection{Comparison of NIHSS Scores between Deteriorating Pa-} tients and Nondeteriorating Patients. A total of 182 patients were included. It was found that 51 cases had a baseline NIHSS score of less than 6 points and 9 cases had END, with an incidence rate of $22.7 \%$. There were 131 cases with a baseline NIHSS score $\geq 6$ and 13 cases with END, and the incidence rate was $10.7 \%$. After intravenous thrombolysis, the NIHSS score of the deteriorating group was greatly higher in contrast to the nondeteriorating group, and it was higher than the baseline NIHSS score of the patients before intravenous thrombolysis, with notable difference, $P<0.05$ (Figure 4).

3.4. CS-MRI Assessing Cerebral Infarction Image. Under the conditions of CS-MRI theory, the patients were analyzed with MRI images. Basal ganglia infarction: infarction of the central branch of the middle cerebral artery and infarction of the blood supply area of the deep perforating artery, as shown in Figure 5. Internal watershed cerebral infarction: infarction at the junction of the cortical branch and deep perforating branch of the middle cerebral artery, as shown in Figure 6.

\subsection{Comparison of Imaging Characteristics between the Two} Groups. A comparison of the number of imaging features between deteriorating patients and nondeteriorating patients revealed that the proportion of basal segment infarction in the deteriorating patient group was much inferior to the nondeteriorating group ( $4.50 \%$ vs. $26.26 \%)$, and the difference was considerable $(P<0.05)$. The proportion of internal watershed cerebral infarction in deteriorating patients was higher relative to nondeteriorating patients ( $40.90 \%$ vs. $6.25 \%)$, and the difference was also considerable $(P<0.05)$. In patients with multisite infarctions, it was found 


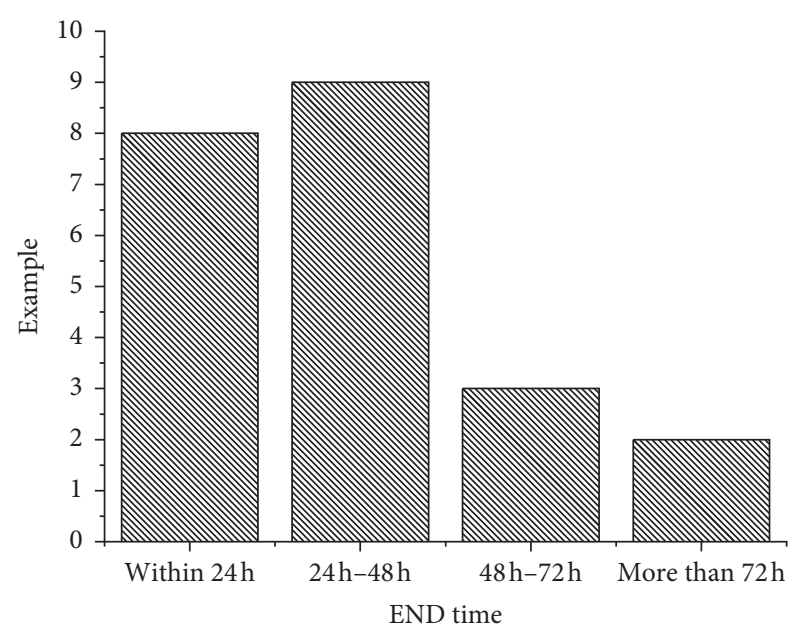

FIGURE 1: END occurrence time of patients.

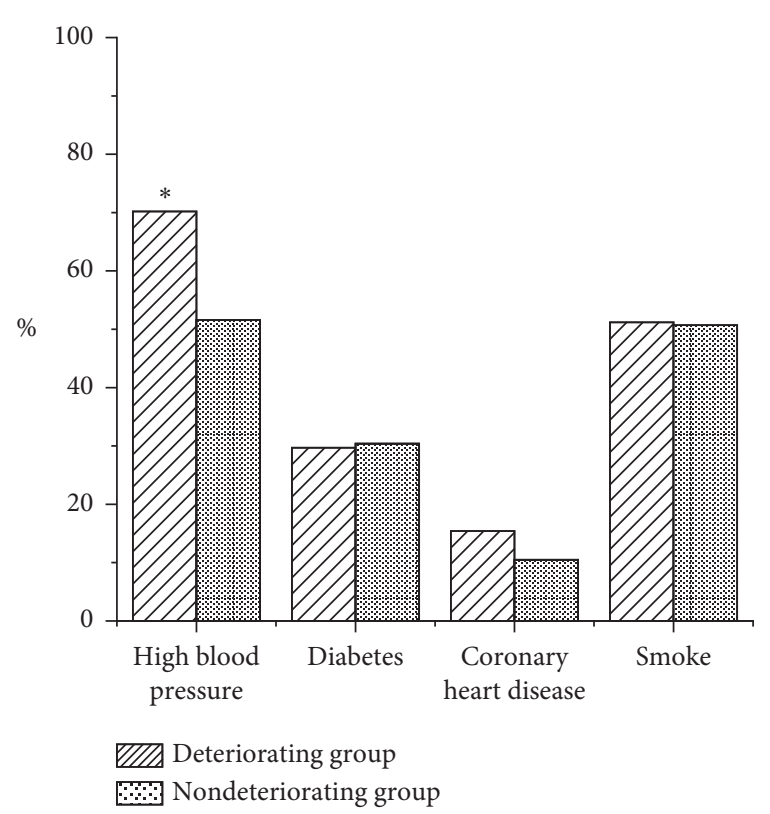

Figure 2: Contrast of patient characteristics between the two groups. Note. ${ }^{*} P<0.05$, the difference was substantial relative to nondeteriorating patients.

that the ratio of data between the two groups was not substantial $(P>0.05)$, as shown in Figure 7.

3.6. Multivariate Logistic Regression Analysis. In univariate analysis, the binary logistic regression analysis model was adopted to analyze variables with $P<0.1$, and then, multiple regression analysis was performed, including NIHSS score and homocysteine. The results in Table 1 showed that, for every increase of NIHSS score: $\mathrm{OR}=0.786,95 \% \mathrm{CI}=0.927 \sim 1.102, \quad P=0.61$; for every increase of $1 \mu \mathrm{mol} / 1$ of homocysteine: $\mathrm{OR}=1.130,95 \%$ $\mathrm{CI}=1.021 \sim 1.062, P=0.035$. The NIHSS score was not correlated with the occurrence of END $(P>0.05)$, and homocysteine was $P<0.05$.

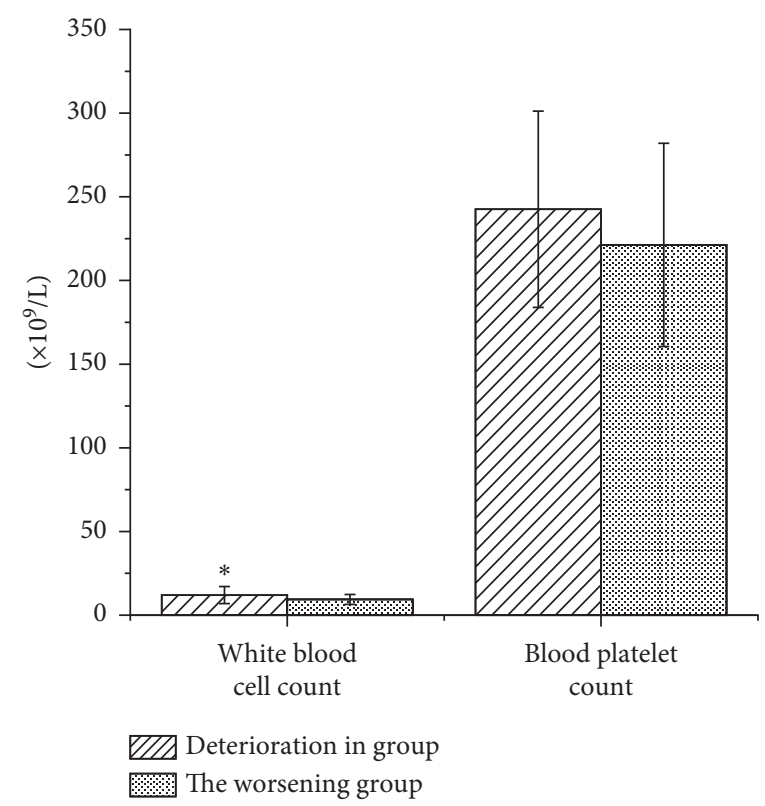

Figure 3: The white blood cell count and platelet count of the two groups of patients. Note. ${ }^{*} P<0.05$, the difference was substantial relative to nondeteriorating patients.

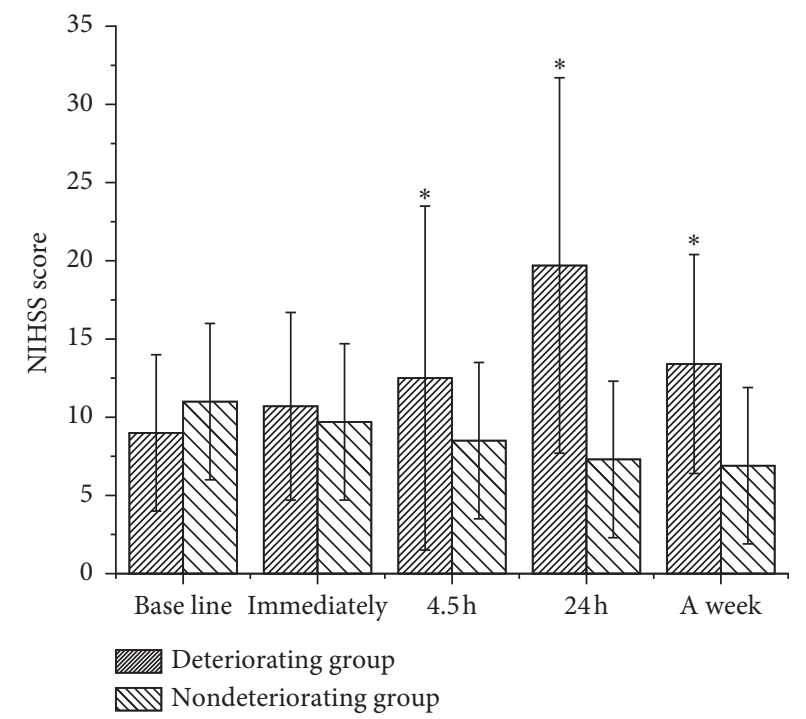

FIGURE 4: NIHSS scores of the two groups of patients at different times. Note. ${ }^{*} P<0.05$, the difference was substantial relative to nondeteriorating patients.

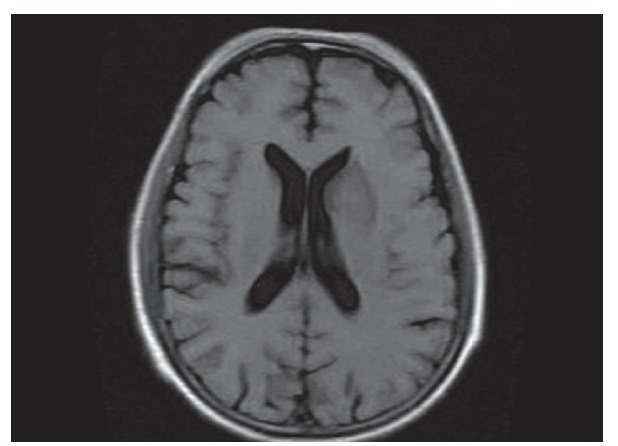

FIgURE 5: Basal ganglia infarction. 


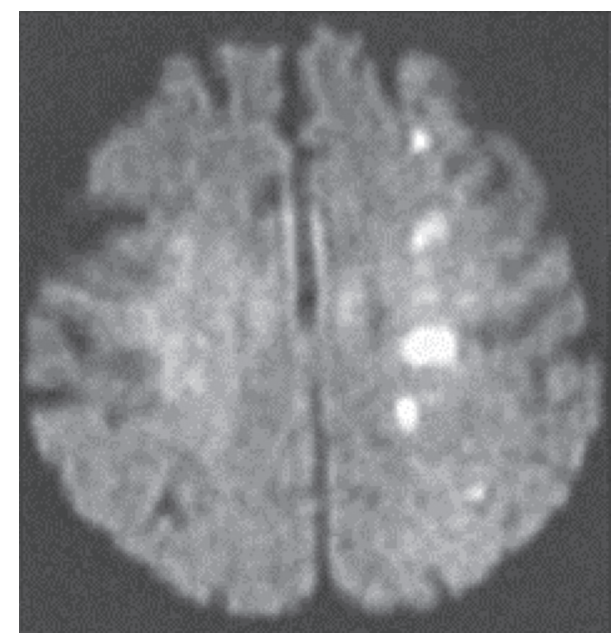

FIgURE 6: Internal watershed cerebral infarction.

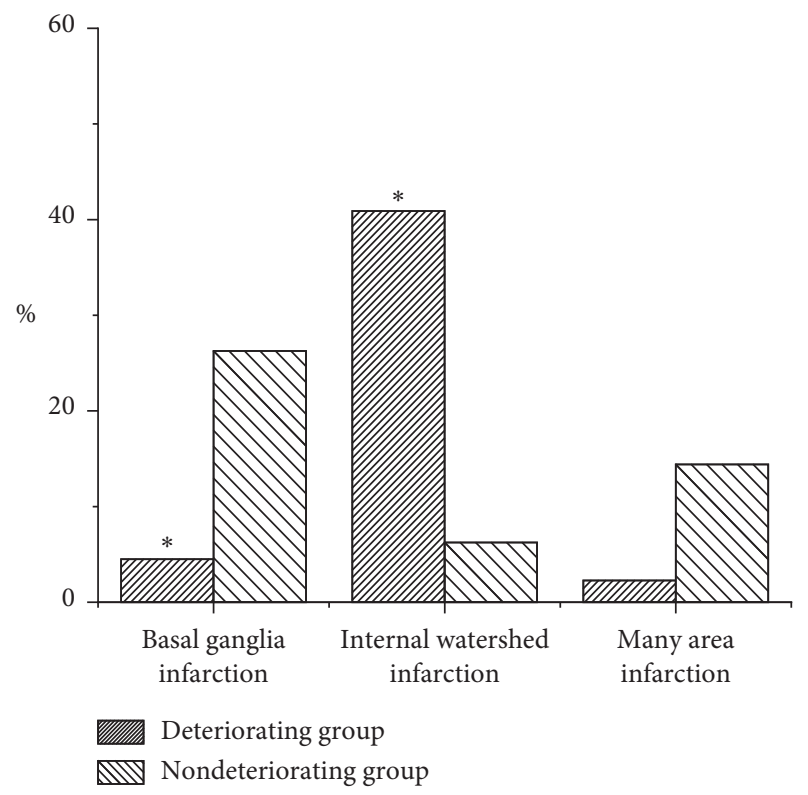

Figure 7: Comparison of the number of infraction sites between the two groups. Note. ${ }^{*} P<0.05$, the difference was substantial relative to nondeteriorating patients.

TABle 1: Logistic regression analysis results of multivariate risk factors.

\begin{tabular}{lcc}
\hline & NIHSS score & Homocysteine \\
\hline OR & 0.786 & 1.130 \\
CI & $0.927 \sim 1.102$ & $1.021 \sim 1.062$ \\
$P$ & 0.61 & 0.035 \\
\hline
\end{tabular}

\section{Discussion}

At present, the aging of population in China is becoming more and more serious, and the diseases related to cerebrovascular are also increasing year by year [8]. According to past data, 160 people died from cerebrovascular diseases in an average of 100,000 people every year, and this number is rising year by year. Cerebrovascular disease is about to become the first fatality rate in China [9]. This type of disease has a high disability rate and fatality rate and poses a huge threat to the lives and health of our people. AIS is one of the most common types of cerebrovascular diseases, accounting for more than half of cerebrovascular diseases [10].

In medicine, intravenous thrombolysis for AIS is the first recommended treatment program internationally, that is, intravenous thrombolysis with recombinant tissue-type plasminogen activator within 4.5 hours of the patient's onset [11]. The occurrence of END after thrombolytic therapy is closely related to the prognosis. Simonsen et al. have pointed out that we should work on the risk factors of END and reduce the risk of END to the lowest level by controlling the risk factors. Moreover, according to the clinical data of different patients, the occurrence of END can be predicted, and corresponding treatment measures can be taken in time. Through endovascular treatment, the damage of END to the patient's nerve function is minimized, so as to improve the prognosis [12]. At the onset of AIS patients, intravenous thrombolytic therapy with alteplase was performed within 4.5 hours. After the thrombolytic therapy, the neurological damage was fluctuating worse than before thrombolysis. A total of 182 patients were included in this study. It was found that 51 cases had a baseline NIHSS score of less than 6 , and 9 cases had END, with an incidence rate of $22.7 \%$. There were 131 cases with a baseline NIHSS score $\geq 6$ and 13 cases with END, and the incidence rate was $10.7 \%$. After intravenous thrombolysis, according to the results, the NIHSS score of the deteriorating group was substantially higher versus the nondeteriorating group, and it was higher than the baseline NIHSS score of the patients before intravenous thrombolysis, $P<0.05$. Obviously, the results of this study were lower than those reported in the literature. It may be that, in the exclusion criteria, the exclusion of patients from bridging therapy reduced the incidence of END in the experiment [13].

Previous studies have found that clinical baseline characteristics: age, gender, diabetes, coronary heart disease, cardiac insufficiency, smoking, drinking, myocardial infarction, history of antiplatelet or lipid-lowering drugs, platelet count, international standardized ratio, low-density lipoprotein, high-density lipoprotein, and fibrinogen were risk factors for cerebrovascular diseases. It may be related to the occurrence of END after intravenous thrombolytic therapy in AIS patients. In the univariate analysis of this study, no correlation was found between these factors and the occurrence of END $(P>0.05)$. Hypertension: long-term hypertension was a risk factor for AIS disease. AIS with higher or lower blood pressure will promote the deterioration of neurological function [14]. NIHSS score: Prochazka et al. pointed out that the baseline NIHSS score was not only an assessment index of neurological impairment but also one of the risk factors for END after thrombolytic therapy [15].

CS-MRI on the head is an effective measure to evaluate cerebrovascular diseases, and the diagnosis of cerebral infarction is more sensitive than CT. Studies have reported that the incidence of basal ganglia infarction in stroke 
patients was lower in deteriorating patients than in nondeteriorating patients, and the incidence of watershed infarction in deteriorating patients was higher than that in nondeteriorating patients [16]. This study found that basal ganglia infarction was more common in the nondeteriorating group, and internal watershed infarction was more common in the deteriorating group.

\section{Conclusion}

CS-MRI was applied to correct the relative error generated by the measurement data and to remove the noise; then, high-quality MRI images can be constructed. After univariate analysis, hypertension, white blood cell count, homocysteine, and NIHSS score were risk factors for END. The binary logistic regression model was adopted for multivariate regression analysis, and it was found that the NIHSS score was not correlated with the occurrence of END, and there was a certain correlation between homocysteine and the occurrence of END. Predicting the occurrence of END through risk factors can actively treat AIS patients with endovascular treatment, which was of great significance to the clinical treatment of patients with AIS in the later stage. The limitation of this study is that when the relationship between the infarct position of the middle cerebral artery supply area and END is studied, excluding severe AIS patients who cannot be evaluated by MRI images after admission will have a certain impact on the results. Moreover, the time taken to evaluate the imaging characteristics of END patients with MRI images is too short and further follow-up of the changes in the prognostic imaging characteristics should be made. In conclusion, the adoption of MRI imaging evaluation provides an approach for the clinical treatment of early neurological deterioration in ischemic stroke after thrombolytic therapy, which has clinical guiding significance.

\section{Data Availability}

No data were used to support this study.

\section{Conflicts of Interest}

The authors declare that they have no conflicts of interest.

\section{Authors' Contributions}

Yiwei Huang and Xiaoyun Sun contributed equally to this work.

\section{References}

[1] Y. Li, W. Zhong, Z. Jiang, and X. Tang, "New progress in the approaches for blood-brain barrier protection in acute ischemic stroke," Brain Research Bulletin, vol. 144, pp. 46-57, 2019.

[2] K. Shkirkova, J. L. Saver, S. Starkman et al., "Frequency, predictors, and outcomes of prehospital and early postarrival neurological deterioration in acute stroke," JAMA Neurology, vol. 75, no. 11, pp. 1364-1374, 2018.
[3] L. M. Tee, E. Y. Kan, J. C. Cheung, and W. C. Leung, "Magnetic resonance imaging of the fetal brain," Hong Kong Medical Journal, vol. 22, no. 3, pp. 270-278, 2016.

[4] M. G. Carneiro, R. Cheng, L. Zhao, and Y. Jin, "Particle swarm optimization for network-based data classification," Neural Networks, vol. 110, pp. 243-255, 2019.

[5] L. Feng, T. Benkert, K. T. Block, D. K. Sodickson, R. Otazo, and H. Chandarana, "Compressed sensing for body MRI," Journal of Magnetic Resonance Imaging, vol. 45, no. 4, pp. 966-987, 2017.

[6] T. Kampf, V. J. F. Sturm, T. C. Basse-Lüsebrink et al., "Improved compressed sensing reconstruction for ${ }^{19} \mathrm{~F}$ magnetic resonance imaging," Magnetic Resonance Materials in Physics, Biology and Medicine, vol. 32, no. 1, pp. 63-77, 2019.

[7] M. E. MacDonald, N. D. Forkert, G. B. Pike, and R. Frayne, "Phase error correction in time-averaged 3D phase contrast magnetic resonance imaging of the cerebral vasculature," PLoS One, vol. 11, no. 2, Article ID e0149930, 2016.

[8] C. Izzo, A. Carrizzo, A. Alfano et al., "The impact of aging on cardio and cerebrovascular diseases," International Journal of Molecular Sciences, vol. 19, no. 2, p. 481, 2018.

[9] Q. Yu, B. Wang, Y. Wang, and C. L. Dai, "Level and trend of cardiovascular disease mortality in China from 2002 to 2016," Zhonghua Xinxueguanbing Zazhi, vol. 47, no. 6, pp. 479-485, 2019.

[10] M. Dunbar and A. Kirton, "Perinatal stroke: mechanisms, management, and outcomes of early cerebrovascular brain injury," The Lancet Child \& Adolescent Health, vol. 2, no. 9, pp. 666-676, 2018.

[11] M. Khazaei, A. Davoodian, M. Taheri, and S. Ghafouri-Fard, "Former antiplatelet drug administration and consequences of intravenous thrombolysis in acute ischemic stroke," Human Antibodies, vol. 28, no. 1, pp. 53-56, 2020.

[12] C. Z. Simonsen, M. L. Schmitz, M. H. Madsen et al., "Early neurological deterioration after thrombolysis: clinical and imaging predictors," International Journal of Stroke, vol. 11, no. 7, pp. 776-782, 2016.

[13] H. J. Irvine, T. W. Battey, A.-C. Ostwaldt et al., "Early neurological stability predicts adverse outcome after acute ischemic stroke," International Journal of Stroke, vol. 11, no. 8, pp. 882-889, 2016.

[14] K. Fujita, K. Tanaka, H. Yamagami et al., "Detrimental effect of chronic hypertension on leptomeningeal collateral flow in acute ischemic stroke," Stroke, vol. 50, no. 7, pp. 1751-1757, 2019.

[15] V. Prochazka, T. Jonszta, D. Czerny et al., "The role of von Willebrand factor, ADAMTS13, and cerebral artery thrombus composition in patient outcome following mechanical thrombectomy for acute ischemic stroke," Medical Science Monitor, vol. 24, pp. 3929-3945, 2018.

[16] C. Ling, X. Fang, Q. Kong et al., "Lenticulostriate arteries and basal ganglia changes in cerebral autosomal dominant arteriopathy with subcortical infarcts and leukoencephalopathy, a high-field MRI study," Frontiers in Neurology, vol. 10, p. 870, 2019. 